\title{
Experimental Study of a Membrane Antenna Surface Adaptive Control System
}

\author{
H. Fang ${ }^{1}$, U. Quijano ${ }^{2}$, V. Bach ${ }^{3}$ \\ Jet Propulsion Laboratory, California Institute of Technology, Pasadena, CA 91109 \\ J. Hill ${ }^{4}$, K. W. Wang ${ }^{5}$ \\ University of Michigan, Ann Arbor, MI 48109
}

\begin{abstract}
Due to their ultra lightweight and high packaging efficiency, membrane reflectors are getting more and more attention for mission architectures that need extremely large in-space deployable antennas. However, maintaining the surface shape of a membrane reflector to the instrument precision requirements is a very challenging problem. This experimental study investigated using PVDF membrane piezoelectric material as actuators to control the surface figures of membrane reflectors. The feasibility of this approach is demonstrated by several sets of test results.
\end{abstract}

\section{Introduction}

SUPPORTED by NASA ESTO ACT program, a technology project has been performed to develop a high $\checkmark$ precision adaptive control architecture for correcting the surface distortions in large membrane antenna reflectors. This technology is developed for future spaceborne earth science remote sensing missions [1, 2]. For high RF frequency applications such Ka-band and w-band, high-precision surface control is necessary for in-space deployable reflectors. Adaptive reflector surface control methods include using temperature gradients [3], boundary control $[4,5]$, cable control [8-9] and etc.

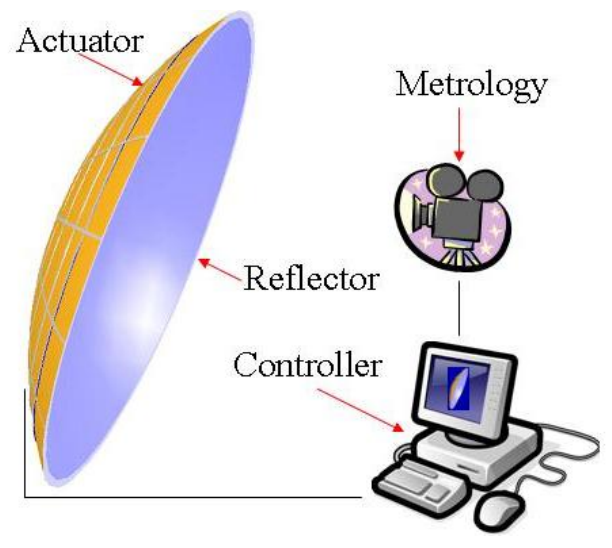

Figure 1. The architecture of a high-precision adaptive control system

As shown in Fig. 1, the architecture of the surface control system discussed by this paper consists of a set of flexible actuators (mounted on the back of a reflector), wavefront sensing metrology subsystem, and active (feedback) controller. Guided by shape control laws, the controller periodically updates voltage signals to control the

\footnotetext{
${ }^{1}$ Technologist III, Mechanical Systems Engineering and Research Division, 4800 Oak Grove Drive, MS 299-100, Senior Member AIAA.

${ }^{2}$ Technologist, Mechanical Systems Engineering and Research Division, 4800 Oak Grove Drive, MS 299-100.

${ }^{3}$ Technologist, Mechanical Systems Engineering and Research Division, 4800 Oak Grove Drive, MS 299-100.

${ }^{4}$ Visiting Scholar, Department of Mechanical Engineering, Ann Arbor, MI 48109.

${ }^{5}$ Stephen P. Timoshenko Professor, Department of Mechanical Engineering, Ann Arbor, MI 48109.
} 
actuator strain at various antenna positions, thus maintaining desired shape contour. The reflectors employed by this study are membrane reflectors. These reflectors include membrane shell reflectors [4] and inflatable reflectors [10]. The control approach discussed by this paper is also applicable thin composite shell reflectors.

To support the development of this adaptive control architecture, several experimental studies have been conducted. These experimental studies include actuator tests, a 0.6-m diameter membrane reflector study and a 2.4$\mathrm{m}$ diameter membrane reflector study. The feasibility of this architecture has been demonstrated not only analytically, but also experimentally by these studies.

\section{Actuator Testing}

To accommodate the packaging and deployment of membrane reflectors, the PVDF (Polyvinylidene fluoride) piezoelectric material is used by this study for the actuators. PVDF is a membrane material that can be rolled up or folded up together with the membrane reflector. Geospace Research Inc. fabricated all single actuators. A single actuator is a piece of PVDF membrane with metallic electrodes on both sides. To increase the actuator force, we tried to combine two actuators together to form a double actuator.

Before assembling the actuators to the membrane reflectors, we conducted actuator tests to investigate the actuator functionality. For these tests, the actuators were adhered onto a 2 mil $(0.051 \mathrm{~mm})$ thick Kapton film. Both single and double actuators were tested using the test set up shown in Fig. 2. The test setup consists of a measuring ruler, supporting structure, voltmeter, and power supply. The power supply was connected to the actuator via flexible circuit to provide the required high voltage.

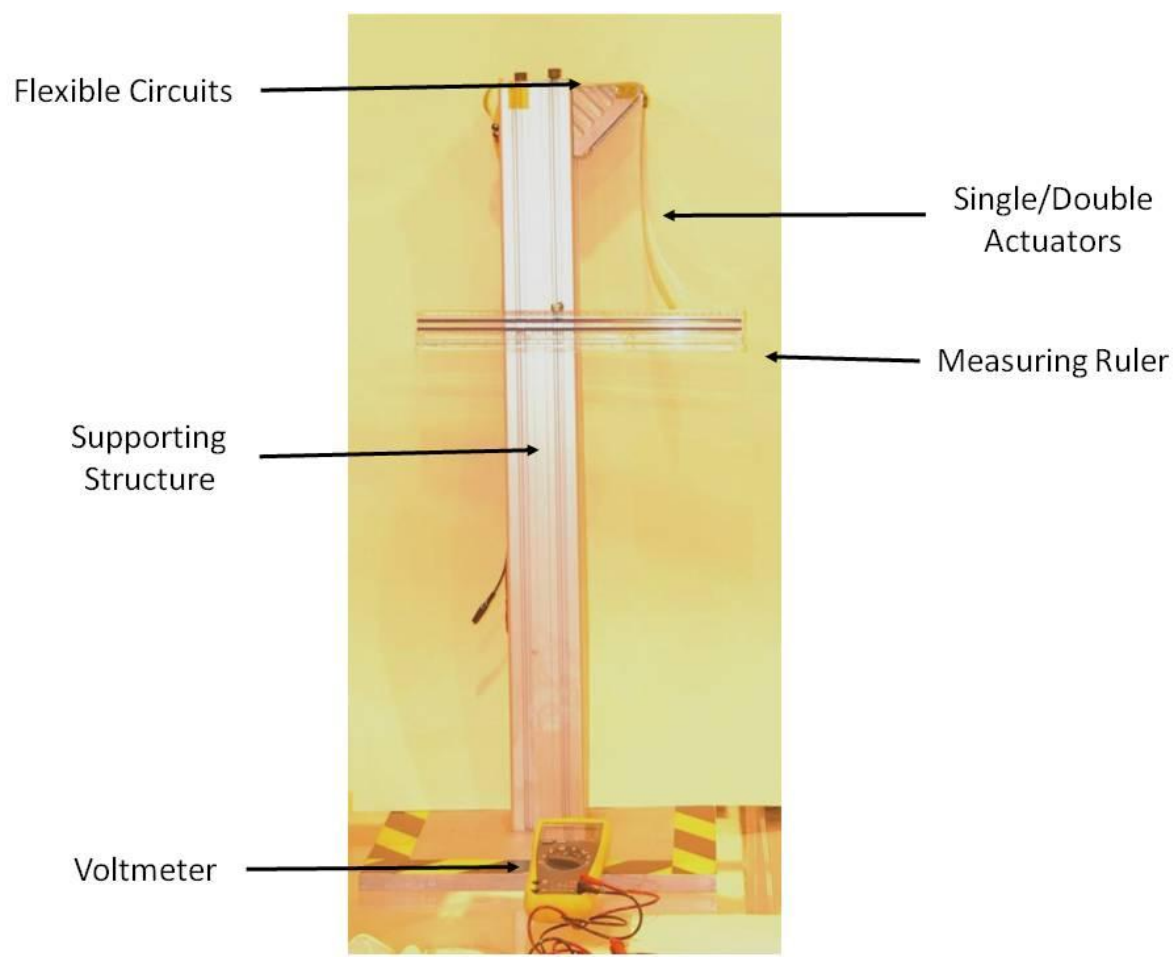

Figure 2. Actuator test setup

For both single and double actuator tests, different voltages ranging from 0 to $-2 \mathrm{kV} /+2 \mathrm{kV}$ were applied to the actuators tested. As increasing negative voltage is applied, the actuator will deflect towards the right. Vice versa, if a positive voltage is applied, the actuator will deflect towards the left. Fig. 3 consists of the data of the voltagedisplacement curve for a single actuator and Fig. 4 is that for a double actuator. 


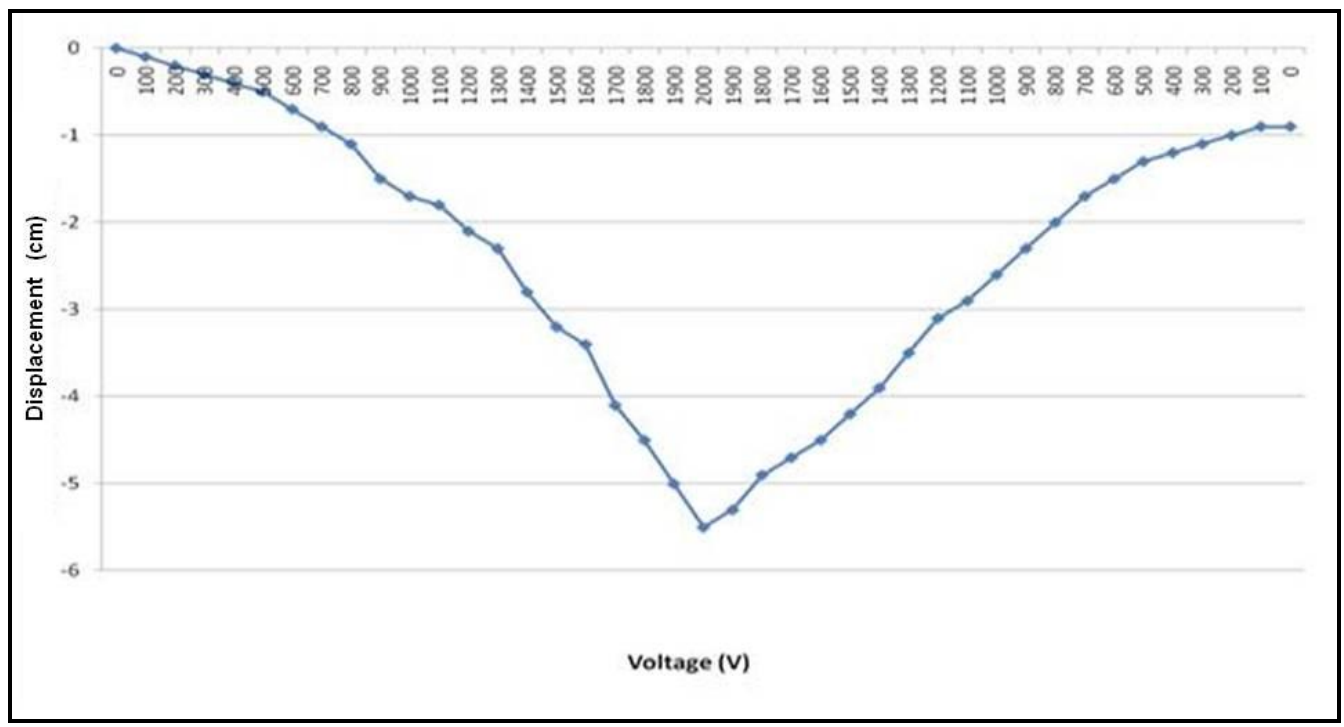

Figure 3. Voltage-displacement curve for a single actuator

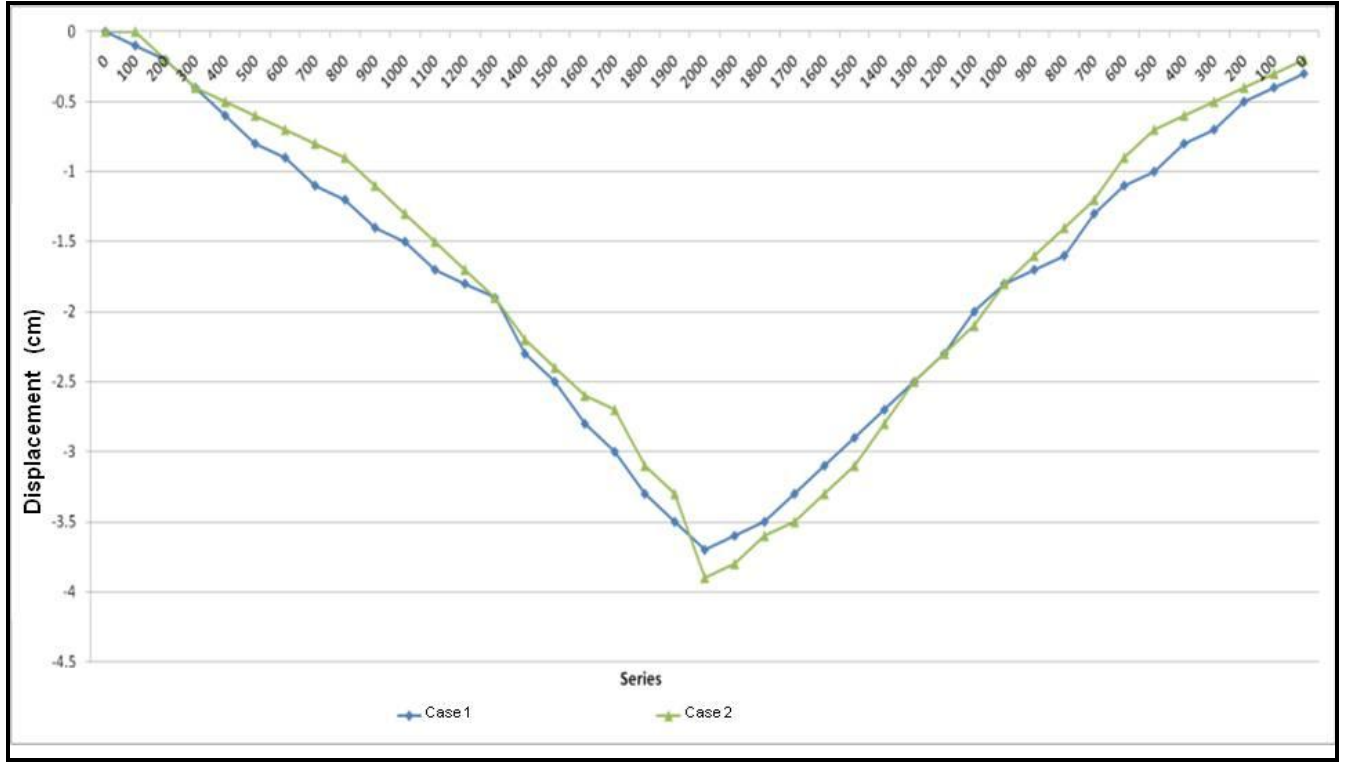

Figure 4. Voltage-displacement curve for a double actuator

It can be seen from Figs. 3 and 4 that function between the voltage and displacement is practically liner. It also can be seen from Fig. 4 that the performance of an actuator is fairly repeatable.

\section{0.6-m Diameter Engineering Models}

As a stepping stone for the fabrication and testing of a 2.4-m diameter reflector, actuators have been attached to several $0.6-\mathrm{m}$ diameter membrane reflectors. The 0.6-m membrane reflectors were fabricated by Mevicon Inc. Double actuators were used for these reflectors and deflection tests were performed in a clean room with a controlled room temperature. A laser displacement sensor is mounted in front of the reflector. It is used to detect the reflector's displacements that are introduced by the actuators. The laser sensor is connected to the multifunctional controller which output the displacement in $\mathrm{mm}$. The DC power supply regulator connects to the high voltage amplifier to provide power to the actuators and also output voltage readings to the Fluke 77III Multimeter. The 0.6-m engineering model test setup is illustrated in Fig. 5. In Fig. 5, 1 is the Kikusui - PAB DC Power Supply Regulator; 2 is the Trek 623B High Voltage Amplifier; 3 is the Fluke 77III Multimeter; 4 is the Keyence LK-GD500 Multifunctional Controller; and 5 is the Keyence LK-G152 Laser Displacement Sensor. 


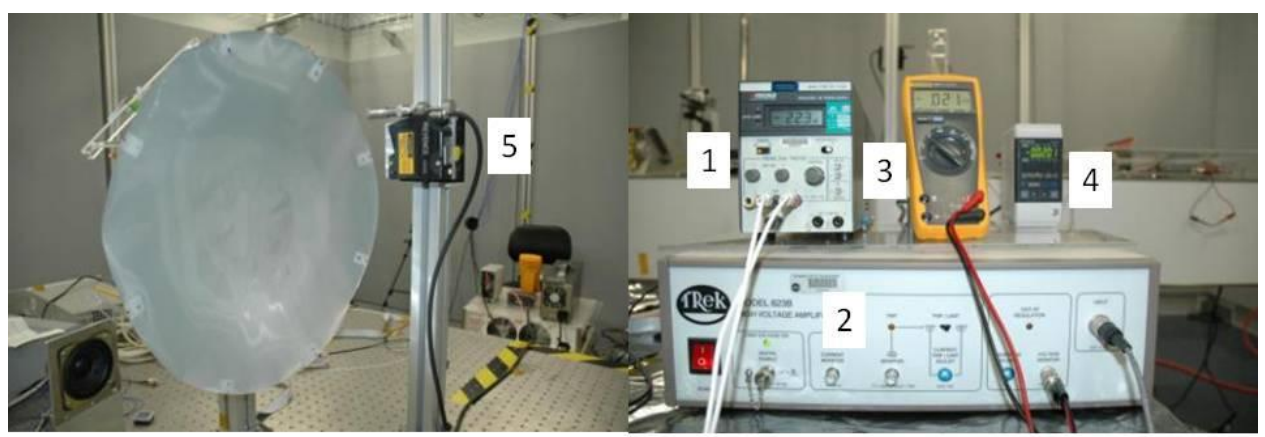

Figure 5. Displacement test setup for a 0.6-m diameter membrane reflector

Besides the laser head measurement, a photogrammetry system was also used to acquire deflections at a number of retro-reflective targets adhered on the back of the reflector. One set of measurement results are presented in Fig. 6.

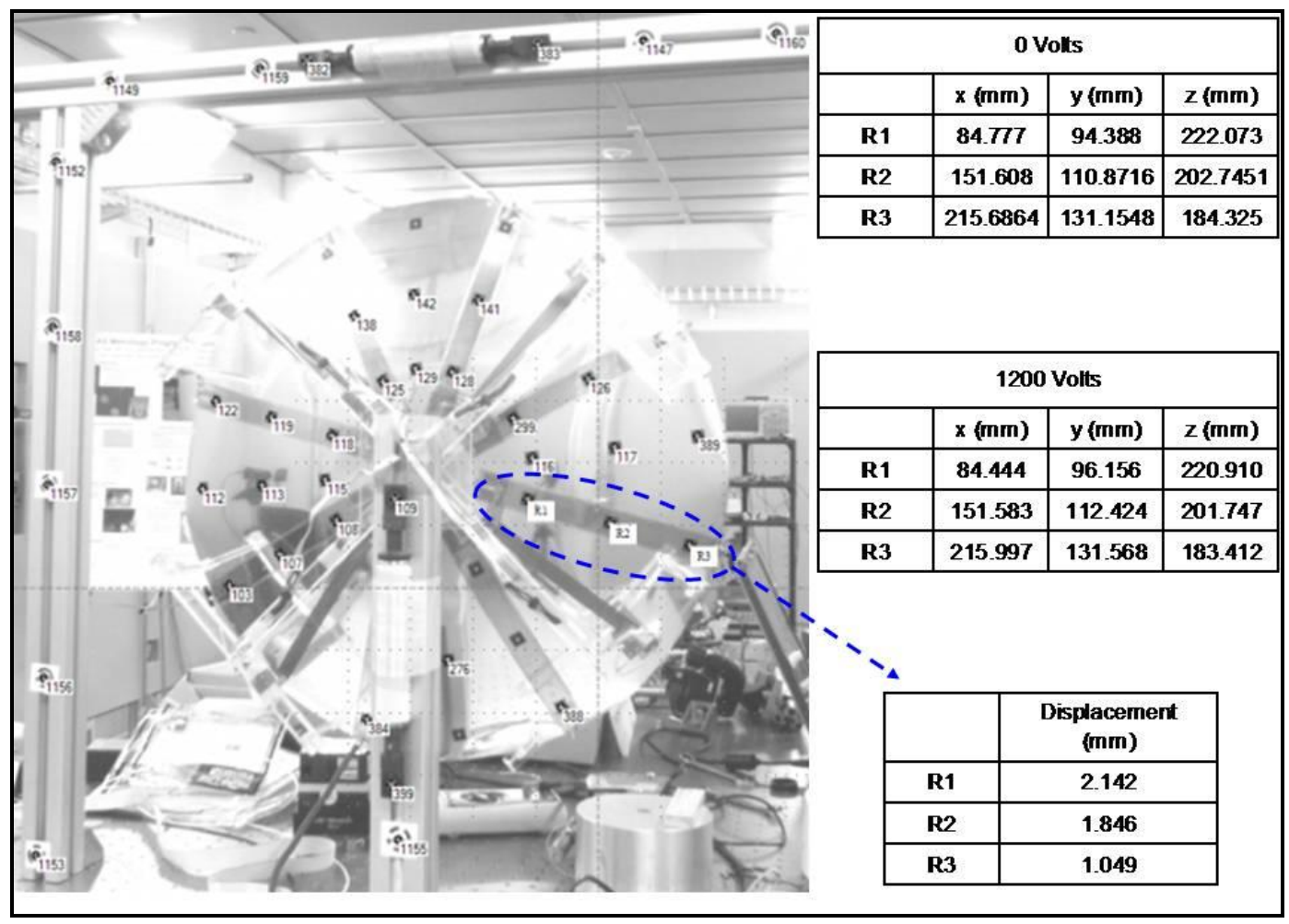

Figure 6. Deformation test results of a 0.6-m reflector

It is concluded from these tests that the approach of assembling the PVDF actuators to membrane reflectors is practical and the deformations introduced by actuators are significant. These results gave us the confidence to work on the 2.4-m diameter engineering model.

\section{2.4-m Diameter Engineering Model}

Fig. 7 illustrates the 2.4 meter engineering model. It is composed of an inflatable membrane reflector, 84 double actuators, 84 single actuators, flexible circuits, and photogrammetry targets. The 2.4-m diameter inflatable membrane reflector was fabricated by NeXolve Corporation. All single actuators are on the left side and all double actuators are on the right side. The laser measurement was set to measure a point at the center of the inflatable reflector and a V-star photogrammetry system was used to measure all the reflective targets. 


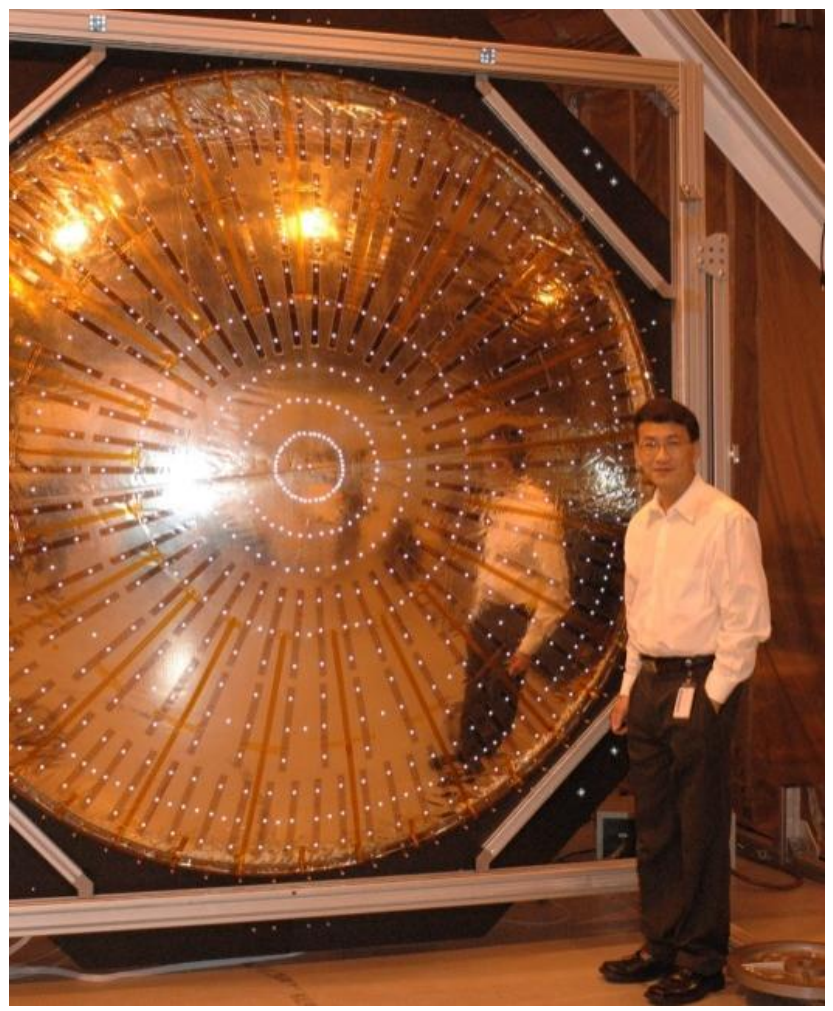

Figure 7. The 2.4 meter engineering model.

Fig. 8 is the close-up view of the engineering model. Flexible circuits are used to bring the voltages to the actuators. Each flexible circuit has 12 traces that can apply voltages to six actuators. Jumpers are used between the actuators and the flexible circuits.

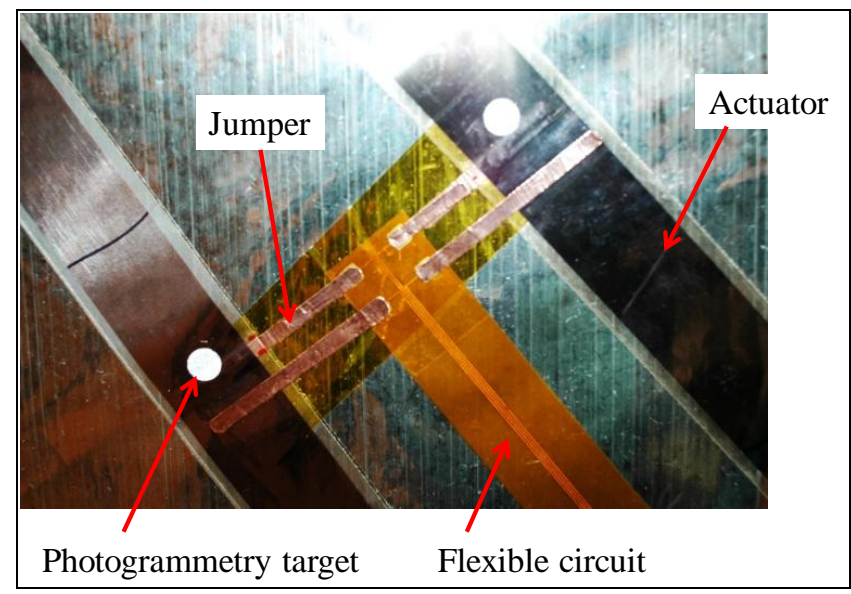

Figure 8. Close up view of the engineering model

Fig. 9 shows the deformation of the reflector while $1000 \mathrm{~V}$ was applied to all single actuators as well as the deformation of the center of the reflector as the function of the voltage. Fig. 10 shows the deformation of the reflector while $1000 \mathrm{~V}$ was applied to all double actuators as well as the deformation of the center of the reflector as the function of the voltage. Fig. 11 shows the deformation of the reflector while different voltages were applied to all the actuators as well as the deformation of the center of the reflector as the function of the voltage. 


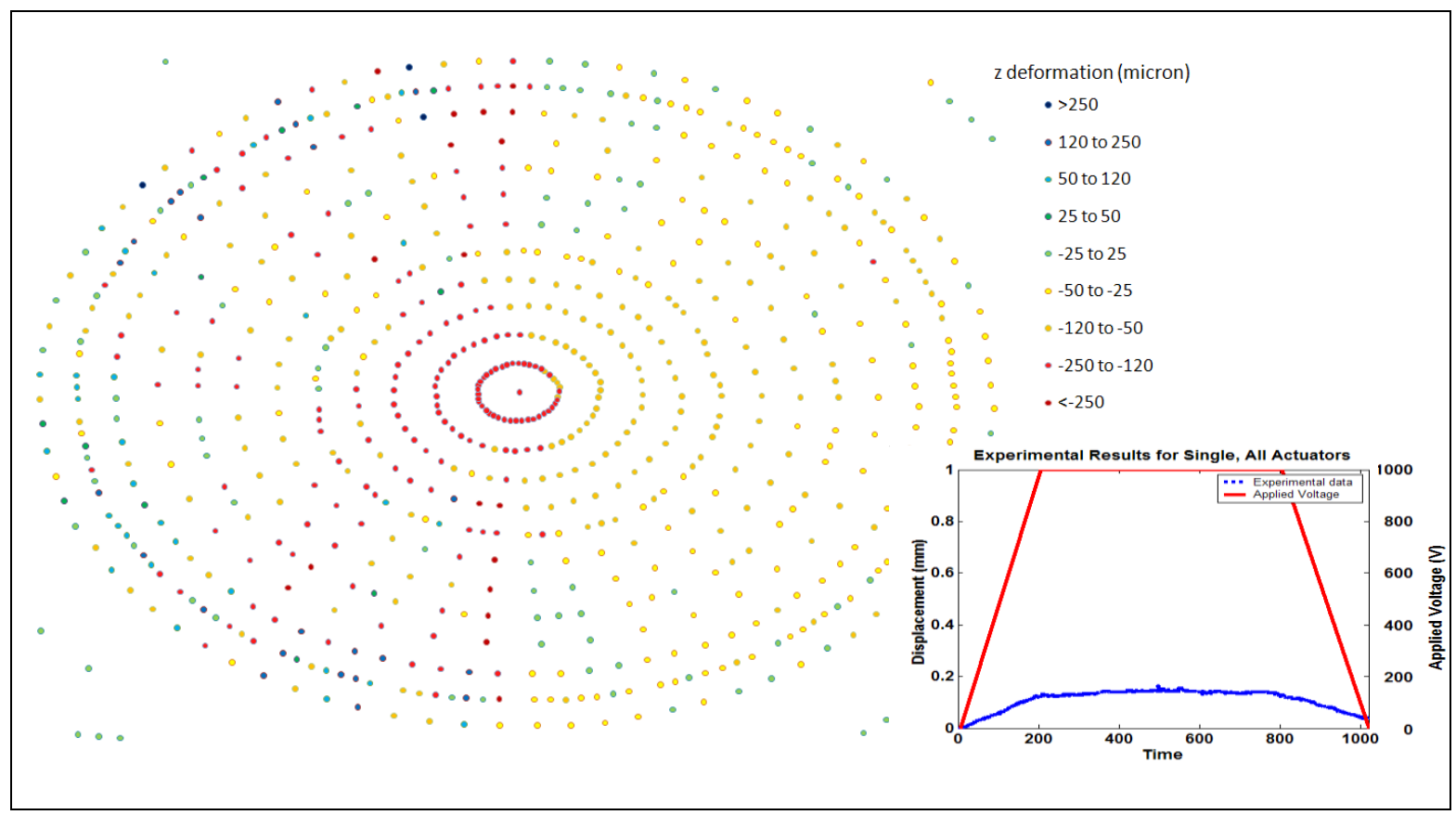

Figure 9. Deformations of the reflector surface and the reflector center point while $1000 \mathrm{~V}$ was applied to all single actuators

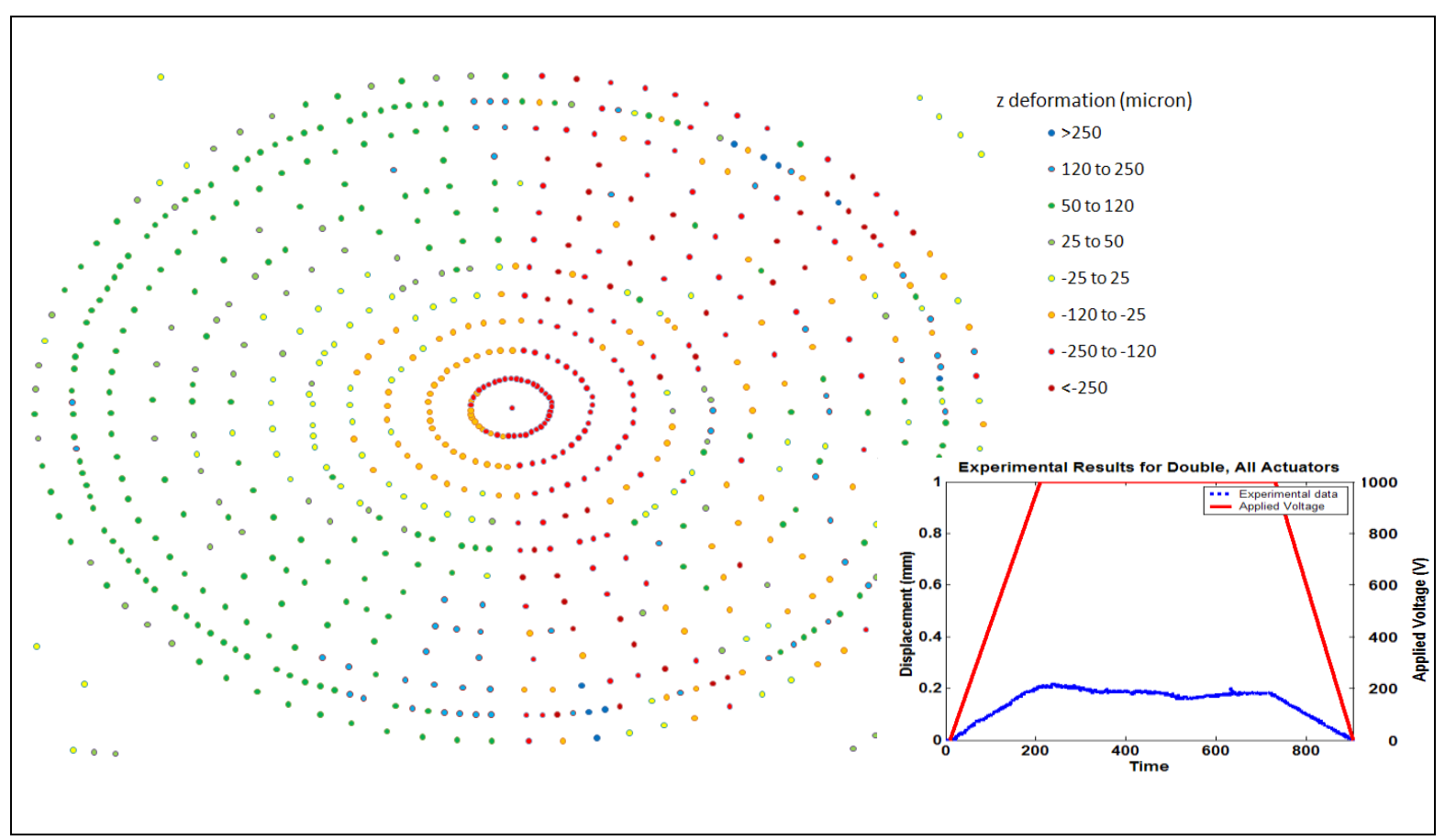

Figure 10. Deformations of the reflector surface and the reflector center point while $1000 \mathrm{~V}$ was applied to all double actuators 


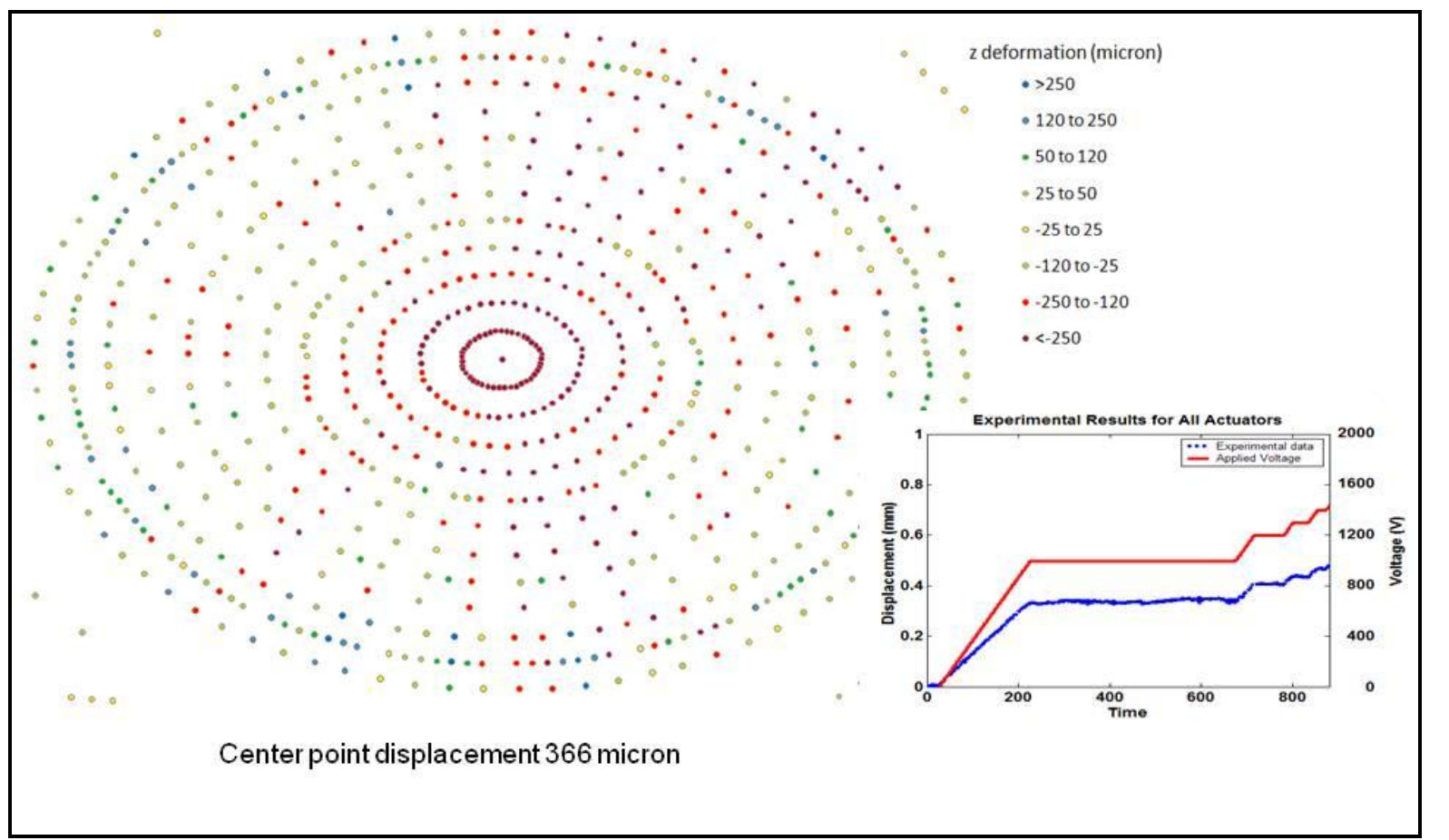

\section{Figure 11. Deformation of the reflector surface and the reflector center point while voltage is applied to all the actuators}

It can be seen from Figs. 9 to 11 that the double actuators introduce more center point deformation than that of single actuators while the same voltages were applied. At 1000 volts, the summation of center point deformations introduced by all single actuators (Fig. 9) and all double actuators (Fig. 10) is almost identical to the same voltage was applied to all the actuators (Fig. 11). While all the actuators were powered to 1000 volts, the center point deforms 366 microns which is significant and sufficient for surface accuracy control with the newly developed low CTE membrane material [11]. It is also observed from this figure that the center deformation and voltage is fairly a linear relationship.

The Influence Coefficient Matrix method and the least squares control law are used for the controller because Influence Coefficient Matrix allows online model identification. The Influence Coefficient Matrix method is based on the principle of superposition: the influences must be linear in order to add them together. The Influence Coefficient Matrix method has been tested and verified on the $2.4 \mathrm{~m}$ reflector with 168 PVDF actuators. This test had following several steps: 1) $1000 \mathrm{~V}$ was applied to half the actuators, and photogrammetry was used to determine the surface deflection; 2) then the surface deflection was measured while $1000 \mathrm{~V}$ was applied to the other half of the actuators; 3 ) finally the surface deflection was measured while 1000 voltage was applied to all the actuators. If the summation of the deflections from step 1 and step 2 equals to the deflection of step 3 , the influence is linear and the Influence Coefficient Matrix method is valid. Fig. 12 and Fig. 13 show the individually tested deflections, the calculated combined deflection, and the measured deflection. Fig. 14 shows the difference between the summation of step 1 and step 2 as well as measured deflection of step 3. It can be concluded from Figs. 12 to14 that the influences are fairly linear and the Influence Coefficient Matrix method is thus verified. 


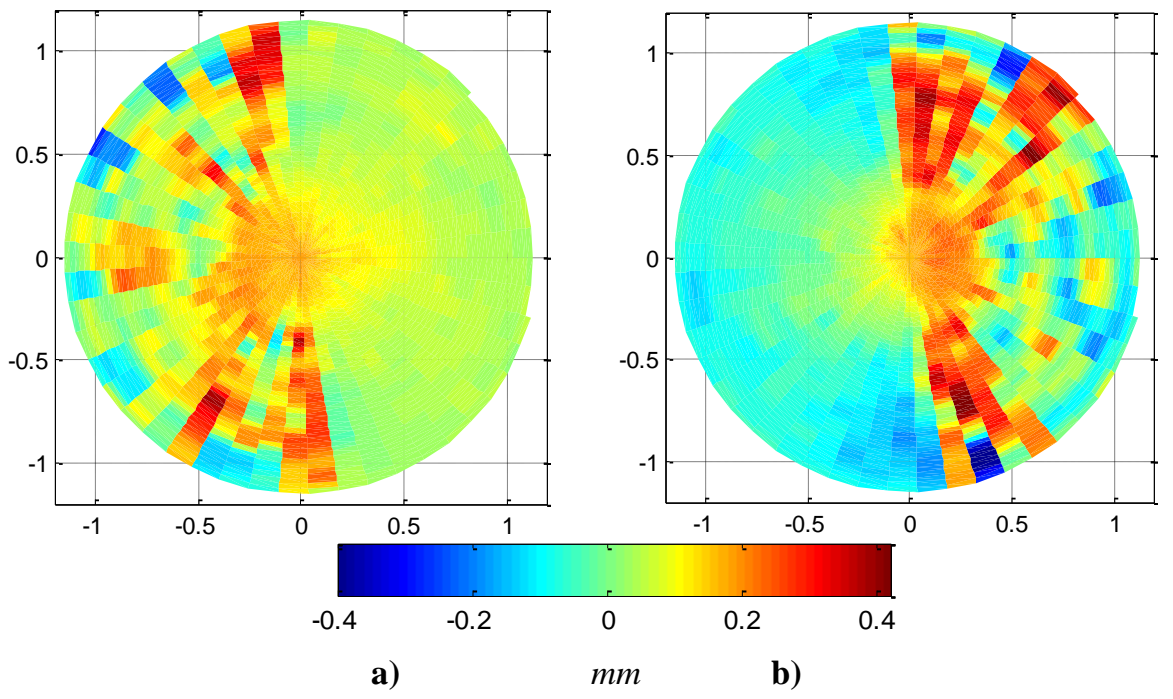

Figure 12. Influence of $\mathbf{1 0 0 0}$ voltage on: a) single actuators, and b) double actuators

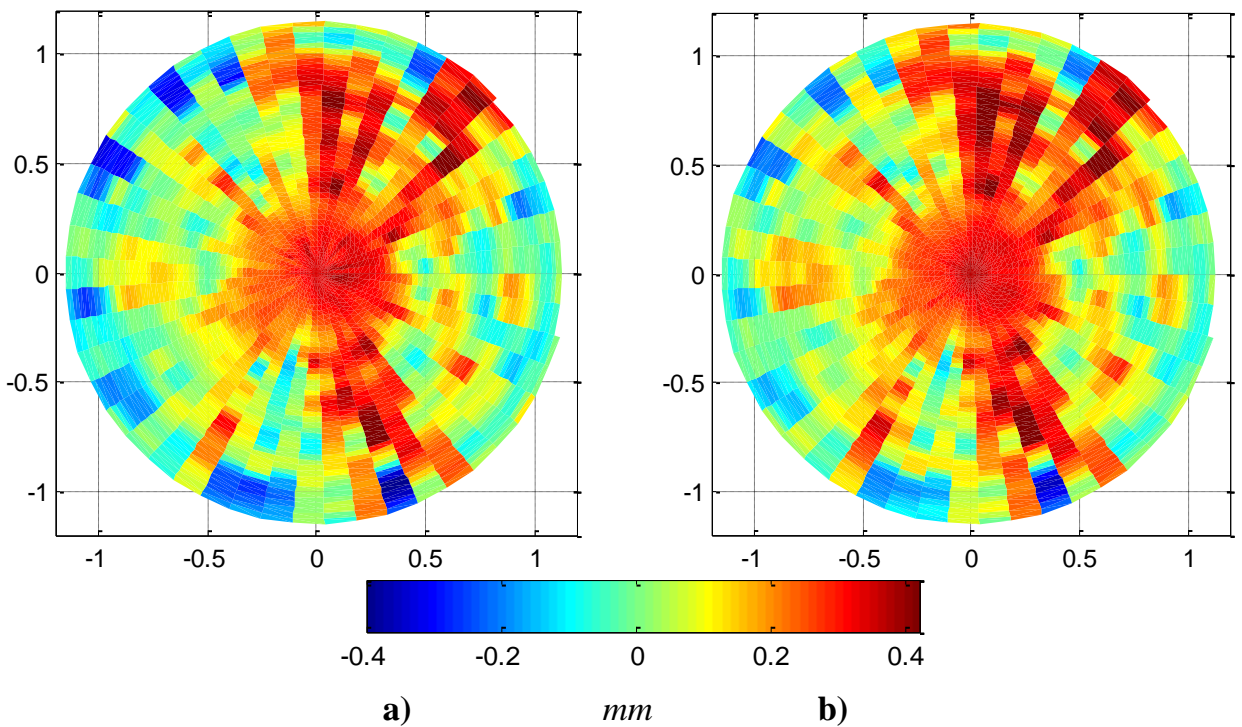

Figure 13. a) Summation of Fig. 10 a) and b), b) measured displacement at $1000 \mathrm{~V}$ 


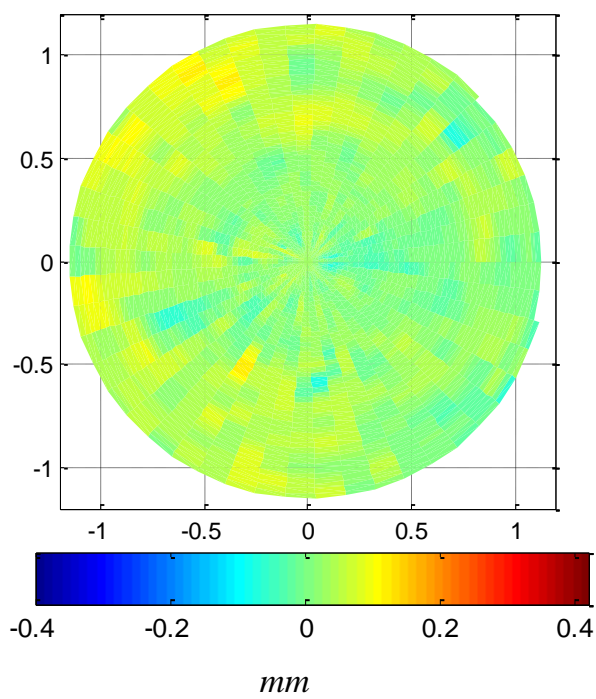

Figure 14. Difference between measured and calculated deflection

\section{Conclusion}

PVDF is a kind of membrane piezoelectric material that can be used to control the surface figures of in-space deployable or inflatable membrane reflectors. After PVDF actuators are bounded to membrane reflectors, they can be rolled up or folded up together with the reflectors. In order to demonstrate the feasibility of using PVDF actuators, several experimental studies have been conducted. The observations from these experimental studies are: 1) the performance of a PVDF actuator is reasonably repeatable; 2) the deformations introduced by PVDF actuators are significant and sufficient; 3 ) the influences from different groups of PVDF actuators are fairly linear and can be superposed on each other, therefore the Influence Coefficient Matrix method and the least squares control law are applicable. It can be concluded based on these observations that the membrane reflector high precision adaptive control architecture discussed by this paper is feasible.

\section{Acknowledgments}

The authors would like to express our appreciations to Frank Djuth of Geospace Research Inc. for developing all the PVDF actuators; to Jim Pearson and Jim Moore for fabricating the 2.4-m inflatable reflector; to Eric Flint of Mevicon Inc. for providing the 0.6-m membrane shells; and to Sean Yoon and Khoa Le of California State University at Los Angeles for helping with the tests. The work described was performed at the Jet Propulsion Laboratory, California Institute of Technology under a contract with the National Aeronautics and Space Administration.

\section{References}

${ }^{1}$ Im, E., Durden, S.L., Rahmat-Samii, Y., Fang, H., Cable, V., Lou, M., and Huang, J., “Advanced Geostationary Radar for Hurricane Monitoring and Studies,” Proc. IEEE Radar Conference, 307-311 (2004).

${ }^{2} \mathrm{Im}$, E., and Durden, S.L., "Instrument Concepts and Technologies for Future Spaceborne Atmospheric Radars," Proc. SPIE Enabling Sensor and Platform Technologies for Spaceborne Remote Sensing, 5659, 61-72 (2005). doi: 10.1117/12.579066.

${ }^{3}$ Haftka, R. T., and Adelman, H. M., "An Analytical Investigation of Shape Control of Large Space Structures by Applied Temperatures,” AIAA Journal, Vol. 23, 450-457 (1985).

${ }^{4}$ Lindler, J. and Flint, E., "Boundary Actuation Shape Control Strategies for Thin Film Single Surface Shells," Collection of Technical Papers AIAA Structures, Structural Dynamics and Materials Conference, Vol. 5, 3360-3370 (2004).

${ }^{5}$ Jenkins, C.H. and Marker, D.K., "Surface Precision of Inflatable Membrane Reflectors," ASME Journal of Solar Energy Engineering, Vol. 120, 298-305 (1998).

${ }^{6}$ Tabata, M. and Natori, M., 1995, “Active Shape Control of a Deployable Space Antenna Reflector," Sixth International Conference on Adaptive Structures, 177-187 (1995).

${ }^{7}$ Jenkins, C. and Schur, W., "Gore/Seam Architectures for Gossamer Structures," Journal of Spacecraft and Rockets, Vol. 39(5), 298-305 (2002). 
${ }^{8}$ Coleman, T. F. and Li, Y., “A Reflective Newton Method for Minimizing a Quadratic Function Subject to Bounds on Some of the Variables," SIAM Journal of Optimization, 6, 1040-1058 (1994).

${ }^{9}$ DeSmidt, H. A., Wang, K. W. and Fang, H., "Optimized Gore/Seam Cable-Actuated Shape Control of Gossamer Reflectors," Journal of Spacecraft and Rockets, Vol. 44(5), 1122-1130 (2007).

${ }^{10}$ Chodimella, S., Moore, J., Otto, J., Fang, H., "Design Evaluation of a Large Aperture Deployable Antenna," AIAA/ASME/ASCE/AHS/ASC Structures, Structural Dynamics, and Materials Conference, Newport, RI, May 1-4, 2006.

${ }^{11}$ J. C. Pearson, J. D. Moore, H. Fang, "Large and High Precision Inflatable Membrane Reflector", AIAA-2010-2500, 51st AIAA/ASME/ASCE/AHS/ASC Structures, Structural Dynamics, and Materials Conference, Orlando, Florida, April 12-15, 2010 